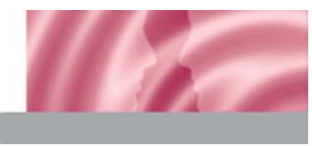

\title{
Using workload measurement tools in diverse care contexts: the experience of staff in mental health and learning disability inpatient settings
}

\begin{tabular}{|r|l|}
\hline Journal: & Journal of Psychiatric and Mental Health Nursing \\
\hline Manuscript ID: & JPM-15-0018.R2 \\
\hline Manuscript Type: & Original Article \\
\hline Keywords: & $\begin{array}{l}\text { Acute Mental Health, Workforce Issues, Staff Perceptions, Service } \\
\text { Management and Planning }\end{array}$ \\
\hline \multicolumn{2}{|c}{} \\
\hline
\end{tabular}

\section{SCHOLARONE ${ }^{\text {w }}$}

Manuscripts 
Abstract

$\underline{\text { Introduction }}$

The critical challenge of determining the correct level and skill-mix of nursing staff required to deliver safe and effective healthcare has become an international concern. It is recommended that evidence-based staffing decisions are central to the development of future workforce plans. Workforce planning in mental health and learning disability nursing is largely under-researched with few tools available to aid the development of evidencebased staffing levels in these environments.

\section{$\underline{\text { Aim }}$}

It was the aim of this study to explore the experience of staff using the Safer Nursing Care Tool (SNCT) and the Mental Health and Learning Disability Workload Tool (MHLDWT) in mental health and learning disability environments.

\section{Method}

Following a 4-week trial period of both tools a survey was distributed via Qualtrics on-line survey software to staff members who used the tools during this time.

\section{$\underline{\text { Results }}$}

The results of the survey revealed that the tools were considered a useful resource to aid staffing decisions; however specific criticisms were highlighted regarding their suitability to psychiatric intensive care units (PICU) and learning disability wards.

\section{Discussion}

This study highlights that further development of workload measurement tools is required to support the implementation of effective workforce planning strategies within mental health and learning disability services. 


\section{Implications for Practice}

With increasing fiscal pressures the need to provide cost-effective care is paramount within NHS services. Evidence-based workforce planning is therefore necessary to ensure that appropriate levels of staff are determined. This is of particular importance within mental health and learning disability services due to the reduction in the number of available beds and an increasing focus on purposeful admission and discharge.

Key words: acute care; evidence-based practice; service management and workforce planning 


\section{Accessible Summary}

\section{What is known on the subject?}

Difficulties with the recruitment and retention of qualified nursing staff have resulted in nursing shortages worldwide with a consequential impact on the quality of care. It is increasingly recommended that evidence-based staffing levels are central to the development of workforce plans. Due to a paucity of empirical research in mental health and learning disability services the staffing needs and requirements for these settings are undefined and the availability of tools to aid staffing decisions is limited.

\section{What this paper adds to existing knowledge?}

This paper provides a valuable insight into the practical uses of these tools as perceived by staff members with day-to-day experience of the requirements of mental health and learning disability wards. It reveals that while workload measurement tools are considered a valuable aid for the development of workforce plans they are limited in their ability to capture all aspects of care provision in these settings. It further emphasises the inapplicability of a oneshoe-fits-all approach for determining nurse staffing levels and the need for individual and customised workforce plans.

\section{What are the implications for practice?}

This study demonstrates that the development of tools for use in mental health and learning disability services is in its infancy and as yet there is no tool that has been validated as such. It highlights the potential for workload measurement tools to aid staffing decisions; however a more holistic approach that considers additional factors is needed to ensure robust workforce planning models are developed for these services. 


\section{Introduction}

The challenge of determining the optimum level and skill-mix of staff required to deliver safe and effective healthcare has become an international endeavour (Gantz et al, 2012). The recruitment and retention of qualified nursing staff has been highlighted as one of the greatest barriers to establishing effective healthcare systems worldwide (Buchan \& Aiken, 2008). Indeed it is estimated that by 2020 a shortage of 600,000 nurses across Europe will be evident following reports that $44 \%$ of nurses from within the UK, Ireland and Poland intend to leave the profession (Gantz et al, 2012). The wide variation in staffing budgets observed across nursing disciplines has led to alterations in staff skill mix including the employment of unqualified staff in place of qualified staff (Bowers \& Flood, 2008; Ryan et al, 2004; Garcia et al, 2005).

This is despite evidence that the quality of nursing care is proportionate to the number of qualified nurses in any given area (Gantz, 2010; Waters, 2003). Indeed international research demonstrates that nurse / patient ratios are directly related to patient mortality rates. For example, Smith (2007), in a study involving 80,000 patients found that wards with a higher registered nurse (RN) proportion (66\% or above) had fewer recorded fatalities. Similarly Spiers' (2005), recorded a dramatic increase in mortality rates in acute medical and surgical wards following an increase in nurse / patient ratios from 1:4 to 1:8. These studies also provide evidence that lower RN proportions are directly linked with adverse events, poor care quality, lowered patient satisfaction levels and diminishing cost effectiveness (Smith et al, 2009).

With increasing longevity and higher levels of patient acuity there is greater demand on nursing workload. Among other issues, factors such as these have long-term effects on care quality and patient outcomes, and have become the focus of international nursing leadership (Duffield et al, 2006; Gantz et al, 2012). The restructuring of services is one such issue 
which presents a global challenge for nurse leaders. In particular the inadequacy of educational budgets to accommodate the retraining of nurses following the dramatic shift towards enhanced care provision and self-management in the community is identified (Gantz et al, 2012). In addition the maintenance of quality and safety standards under increasing budget constraints is identified as particularly challenging, further exacerbated by the continuing media focus on patient safety incidents and poor quality care (Gantz et al, 2012).

\section{UK Context}

In the UK, the recent inquiry into the appalling care of patients at the Mid-Staffordshire hospital revealed that inadequate staffing levels, recruitment, and training were a primary contributor to the 'declining professionalism' and 'tolerance of poor standards' uncovered (Francis, 2013, p.45). In response to public demand for the immediate overhaul of patient care, Compassion in Practice $(\mathrm{DH}, 2012)$ was published providing a strategy for the enhancement of overall care provision in all care and support settings across England. The aim of the document is to ensure services take the steps required to establish evidence based staffing levels and redesign their workforce plans with staff skill mix as a central imperative (DH, 2012; Munro \& Baker, 2007).

It recommends that, staff numbers and skill-mix; professional judgement and scrutiny; local and contextual factors; a multi-professional approach; and openness and transparency, are central to the development of staffing models (NQB, 2013). It further emphasises the need to utilise evidence-based tools to ensure that 'patient care needs and expert professional opinion' form the basis of any future workforce plans (NQB, 2013, p.18). Within the document a number of tools are indicated for use in specific care contexts such as, Birthrate Plus, the Paediatric Acuity and Nursing Dependency Assessment (PANDA) Tool and the Safer Nursing Care Tool (Hurst, 2003; Harrison, 2004; Hurst, 2008; Hurst, 2010, Shelford Group, 2014). However, the immediate need for tools and approaches that are tailored to 
complex settings such as mental health, learning disability and community services is highlighted (DH, 2012; NQB, 2013).

\begin{abstract}
National Project
In May 2013, a national project was initiated that aimed to improve existing understanding of the staffing needs and requirements of mental health $(\mathrm{MH})$ and learning disability (LD) services. A primary objective was to identify and evaluate evidence-based tools currently in use. A review of extant literature revealed that the development of robust models for determining nursing workloads in these settings is a common theme (Happell, 2008). Research evidence highlights the association between increased or excessive workloads and increased levels of emotional exhaustion in mental health nurses (Tummers et al, 2001). In addition research indicates that higher numbers of qualified nurses are associated with decreased mortality rates and lower rates of seclusion (Smith et al, 2009).
\end{abstract}

Negative associations between lower numbers of qualified nurses and the development of effective therapeutic patient / practitioner relationships are also highlighted (Hoekstra, Lendemeijer \& Jansen, 2004). The review also revealed that reliable and valid evidencebased tools are limited both nationally and internationally. Although some researchers advocate their use, criticisms include their tendency to underestimate the time needed to establish therapeutic relationships; thereby reducing nursing to a task orientated endeavour (Happell, 2008). In addition some researchers suggest that they are unable to accurately calculate workload in mental health due to their inability to account for fluctuating acuity between shifts or episodes of treatment (Wendling, 2003).

In order to identify tools currently being used in the UK an informal enquiry was circulated via the Nurse Directors Network. It was discovered that the Safer Nursing Care Tool (SNCT) (Shelford Group, 2014), developed for general nursing had recently been adapted for use in 
mental health and learning disability inpatient wards. The SNCT is highly regarded for its comprehensive approach and demonstrates good validity and reliability in acute care settings (NQB, 2013); however reliability and validity testing for MH and LD settings is ongoing. Patient dependency definitions and workload equivalence estimates used within this tool are based upon data gathered in UK quality wards ensuring the exclusion of substandard wards (Hurst, 2010). It is therefore currently only relevant for use in the UK (Smith et al, 2009).

A second calculation tool the Mental Health and Learning Disability Workload Tool (MHLDWT) was identified via the forum. The MHLDWT was developed during the Nursing and Midwifery Workload and Workforce Planning (NMWWP) programme in Scotland (Lockhart et al, 2010) and mandated for use in mental health and learning disability inpatient services (Kellagher et al, 2010). It has not been validated against nationally derived benchmarks: however it adopts a comprehensive and triangulated approach. It is suggested that both tools are costly to set up and require the commitment of staff and financial support.

\begin{abstract}
Aims
In view of the limited availability both nationally and internationally of customised tools for use in mental health (MH) and learning disability (LD) inpatient services the present study aimed to explore the usability of both the SNCT and MHLDWT within these settings from the perspectives of staff.
\end{abstract}

\title{
Objectives
}

The study objectives were to capture the experience of staff using the tools in different clinical environments in order to make recommendations about their suitability for use in mental health and learning disability inpatient settings. The data gathered during the study would subsequently be used to inform the development of national staffing guidelines for 
mental health and learning disability services in accordance with the overall objectives of the national project.

\section{Method}

\section{Participants and Procedure}

Six UK NHS mental health and learning disability trusts agreed to trial both tools over a 4week period. Participating trusts were asked to include Adult Acute (AA), Older Adult (OA), Psychiatric Intensive Care Unit (PICU), and Learning Disabilities (LD) wards only in the trial. Forensic Wards and Mother and Baby Units were excluded due to limited access to these ward types and the variation with which these services are delivered. Each trust nominated a lead contact responsible for the co-ordination of the 4-week trial. A one-day training workshop was delivered during which demonstrations of both tools were provided along with customised templates to record all ward data. Lead contacts were asked to return the completed templates at the end of the 4-week period. In addition they were asked to distribute a Qualtrics online survey to all individuals involved with trial use of the tools during the 4-week period.

\section{Materials}

Workload Measurement Tools

The SNCT (Shelford Group, 2014), shown in Figure 1, is a workload (acuity) quality measure that uses a sophisticated algorithm to calculate workload based upon occupancy, throughput, patient dependency, direct patient care times and ward overhead data (NQB, 2013). The MHLDWT (NMWWP, 2013), shown in Figure 2 is a timed-task activity measure that utilises information about daily tasks and activities to calculate the typical workload of a ward. Both tools are presented in an Excel spread-sheet format and feature separate tabs for Workload and Professional Judgement (PJ) calculations. Workload calculations for 
the SNCT are based upon patient care dependency levels (1 through $4 a)^{*}$ categorised by staff members. Workload calculations for the MHLDWT are based upon tasks associated with the delivery of inpatient care across four separate areas: admission \& discharge; patient specific; task specific; group-work. 
Figure 1. Safer Nursing Care Tool (SNCT)

Workload Calculator

\begin{tabular}{|lll|l|}
\hline Element & Your Ward & Your Ward & Benchmark \\
Dep 1 patients (daily average)? & 7 & $32 \%$ & $35 \%$ \\
Dep 2 patients (daily average)? & 6 & $27 \%$ & $29 \%$ \\
Dep 3 patients (daily average)? & 6 & $27 \%$ & $27 \%$ \\
Dep 4a patients (daily average)? & 2 & $9 \%$ & $9 \%$ \\
4b Spcld patients (daily average)? & 1 & $5 \%$ & $4 \%$ \\
Patients & 22 & $100 \%$ & 21.1 \\
\hline Preferred time-out? & $24.0 \%$ & & \\
Preferred RforA time? & $0.0 \%$ & $58 \%$ & \\
Preferred RN proportion? & 17.3 & & \\
\hline RNs required & 12.5 & 29.8 & \\
HCAs required & 2.8 & \\
Total FTEs required & & \\
\hline
\end{tabular}

Care Dependency Categories

\begin{tabular}{|c|c|}
\hline Level & Description \\
\hline Dep. 1 & $\begin{array}{l}\text { Self-caring patients who can do most daily-living activities unaided. Minimal therapeutic care is needed. Likely to be a recovering } \\
\text { patient about to go home. }\end{array}$ \\
\hline Dep. 2 & $\begin{array}{l}\text { More dependent on ward staff for his/her personal care and safety. S/he may be able to complete some daily-living activities unaided. } \\
\text { Previously close observation and therapeutic care is tailing off. }\end{array}$ \\
\hline Dep. 3 & $\begin{array}{l}\text { III and heavily reliant on the ward team for her his/her safety and care. It's likely that s/he is agitated, unstable and unpredictable, } \\
\text { posing a threat to him/herself and others. Close observation and therapeutic support are usually required. Could be 'sectioned'. }\end{array}$ \\
\hline Dep. 4 & $\begin{array}{l}\text { Desperately ill and dependent on the ward team for his/her care, safety and welfare. Highly likely to abscond, self-harm or injure } \\
\text { others. Close observation and therapeutic attention is likely to feature heavily in the care plan. Probably 'sectioned'. }\end{array}$ \\
\hline Dep. $4 b$ & 'Specialed' patients - requiring unbroken, one-to-one supervision by one or more staff. \\
\hline
\end{tabular}


Professional Judgement Calculator

\begin{tabular}{|c|c|c|c|c|c|c|c|c|c|c|c|c|c|}
\hline \multicolumn{14}{|c|}{ For the Professional Judgement method use this template } \\
\hline \multirow[t]{2}{*}{ Row } & Column B & C & D & $\mathbf{E}$ & $\mathbf{F}$ & G & $\mathbf{H}$ & I & $\mathrm{J}$ & K & $\mathbf{L}$ & M & $\mathbf{N}$ \\
\hline & \multicolumn{6}{|l|}{ Seven-Day Wards } & Mon & Tues & Wed & Thur & Fri & Sat & Sun \\
\hline 1 & \multicolumn{6}{|c|}{ N.B. Italicised red values can be changed by you } & & & & & & & \\
\hline 2 & \multicolumn{6}{|c|}{ Enter the length of your early or day shift in this row } & 7.5 & 7.5 & 7.5 & 7.5 & 7.5 & 7.5 & 7.5 \\
\hline 3 & \multicolumn{6}{|c|}{ Enter the number of nurses on early or day duty in this row } & 4 & 4 & 4 & 4 & 4 & 3 & 3 \\
\hline 4 & \multicolumn{6}{|c|}{ If you have a three-shift system then enter the length of your late duty in this row } & 7.5 & 7.5 & 7.5 & 7.5 & 7.5 & 7.5 & 7.5 \\
\hline 7 & \multicolumn{6}{|c|}{ Enter the number of nurses on night shift duty in this row } & 3 & 3 & 3 & 3 & 3 & 2 & 2 \\
\hline 8 & \multicolumn{6}{|c|}{ What is your sickness and absence level? The average is $22 \%$, entered as 0.22 in cell $\mathrm{H} 8$} & $22 \%$ & & & & & & \\
\hline 9 & \multicolumn{3}{|c|}{ Result: staff needed is: } & 18.9 & FTE & Hours $=$ & 109.8 & 109.8 & 109.8 & 109.8 & 109.8 & 79.3 & 79.3 \\
\hline
\end{tabular}


Figure 2. Mental Health and Learning Disability Workload Tool (MHLDWT)

Workload Calculator

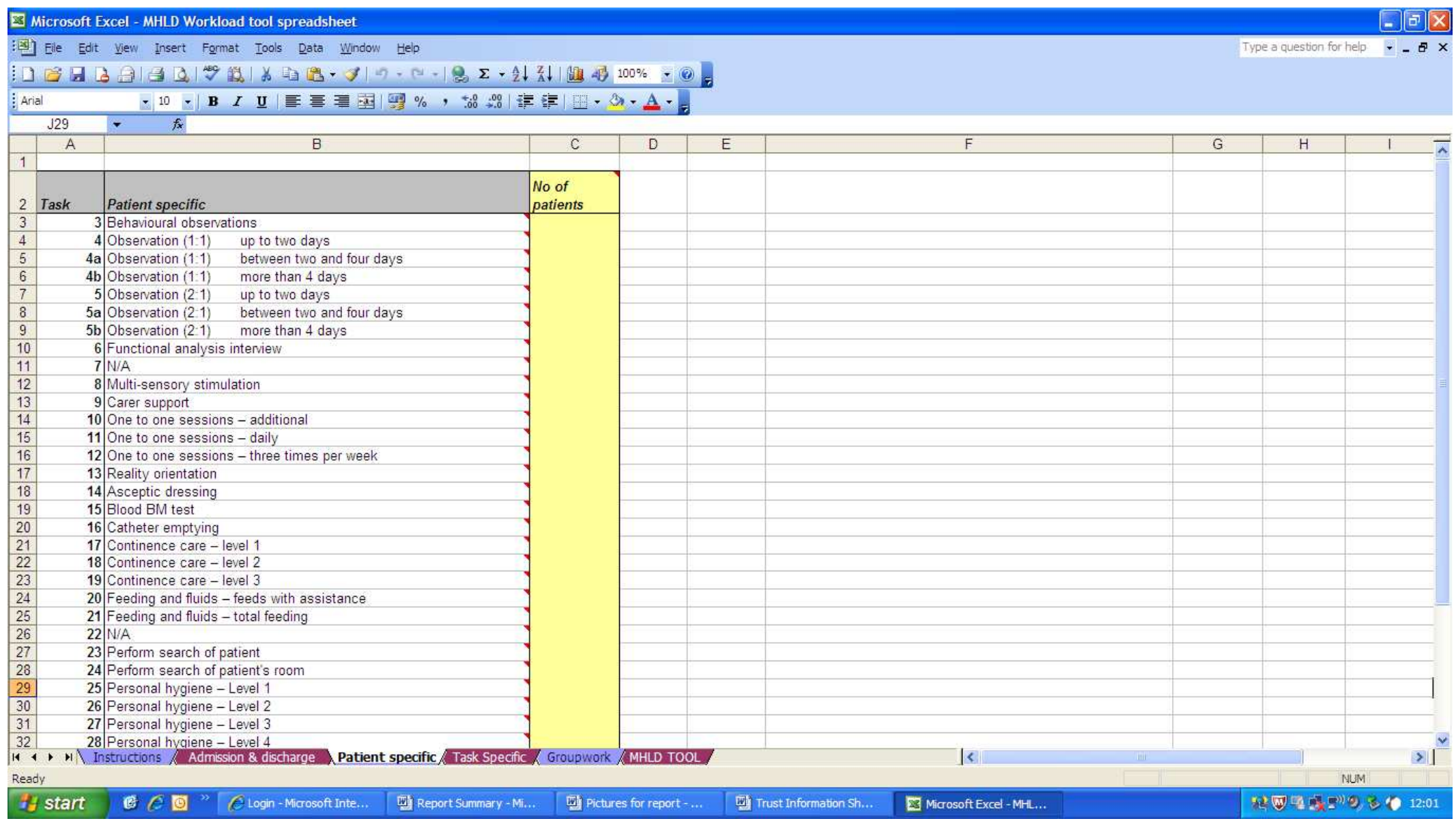


Professional Judgement Calculator

\begin{tabular}{|c|c|c|c|c|c|c|c|c|c|c|}
\hline \multicolumn{3}{|c|}{ WEEK 2 - TOTAL WTE'S } & & & \multicolumn{6}{|c|}{ WEEK 2 - AGREED SKILL MIX } \\
\hline RN WTE = & & 0.00 & & & 7 & 6 & 5 & 4 & 3 & 2 \\
\hline NA WTE = & & 0.00 & & & 0.00 & 0.00 & 0.00 & 0.00 & 0.00 & 0.00 \\
\hline NN WTE = & & 0.00 & & & & & & & & \\
\hline \multicolumn{4}{|c|}{ AVERAGE 2 WEEK WTE'S } & & \multicolumn{6}{|c|}{ AVERAGE TOTAL AGREED SKILL MIX FOR 2 WEEK PERIOD } \\
\hline & Week 1 & Week 2 & Average & & 7 & 6 & 5 & 4 & 3 & 2 \\
\hline RN WTE = & 0.00 & 0.00 & 0.00 & Week 1 & 0.00 & 0.00 & 0.00 & 0.00 & 0.00 & 0.00 \\
\hline NA WTE = & 0.00 & 0.00 & 0.00 & Week 2 & 0.00 & 0.00 & 0.00 & 0.00 & 0.00 & 0.00 \\
\hline NN WTE $=$ & 0.00 & 0.00 & 0.00 & AVERAGE & 0.00 & 0.00 & 0.00 & 0.00 & 0.00 & 0.00 \\
\hline
\end{tabular}


On-line Survey

The first section of the Qualtrics on-line survey concerned ethics, consent and withdrawal of participation; the remaining sections featured Likert items and open-ended questions. Participants were specifically asked to rate their level of agreement from 1 (strongly disagree) to 6 (strongly agree) with statements regarding the usability of both tools. In addition they were given a series of open-ended questions regarding the usability of the tools and their suitability to differing clinical environments.

\section{Data Analysis}

Descriptive statistics were generated via Qualtrics survey software. All qualitative data was exported and analysed manually using thematic analysis as outlined by Brown \& Clarke (2008).

\section{$\underline{\text { Results }}$}

\section{Demographics}

A total of 14 completed surveys out of a possible 20 were returned ${ }^{1}$. Four $(29 \%)$ of the surveys returned were from AA wards, four $(29 \%)$ were from OA wards, three $(21 \%)$ were from PICU wards and three (21\%) were from LD wards

\section{Safer Nursing Care Tool (SNCT)}

Eleven (79\%) respondents agreed that the SNCT is practical, easy to use, and suitable for calculating staffing requirements in their clinical area. In addition, 10 (71\%) respondents agreed that when balanced against their professional judgement the SNCT is a valuable resource for workforce planning. Qualitative comments revealed that some staff felt the tool was limited in its ability to capture 'all activities carried out by staff' due to its focus on patient

\footnotetext{
${ }^{1}$ Response rate of $70 \%$
} 
1

2

3

4

5

6

7

8

9

10

care dependency levels. Tasks that were highlighted as having a contribution to workload without relating directly to the clinical care of patients are presented in Table 1. It was also revealed that some participants viewed the process of categorising patients according to 'care dependency' levels ( 1 through $4 a$ ) as potentially problematic. More specifically it was suggested that the person selected for this task must have knowledge of the daily clinical needs of patients. This knowledge was deemed to be specific to certain bands of nurses 'as a band 6 I am not necessarily as up to date with 14 individuals care and treatment as the regular band 5 nurse due to different work commitments/missing handovers'.

Table 1. A table to illustrate tasks that contribute to workload but are not directly related to patient care dependency level as perceived by participants

\begin{tabular}{|l|l|}
\hline \multirow{4}{*}{ Administrative } & Task \\
\cline { 2 - 2 } & Mental health assessment reports \\
\cline { 2 - 2 } & Patient admission \\
\cline { 2 - 2 } & Patient transfer \\
\cline { 2 - 2 } & Patient discharge \\
\cline { 2 - 2 } & Updating assessment care plans \\
\hline Othection control & Cleaning equipment \\
\cline { 2 - 2 } & Schedules \\
\cline { 2 - 2 } & Checking ward environments \\
\hline & Responding to emergencies \\
\cline { 2 - 2 } & De-escalation processes \\
\hline
\end{tabular}




\section{MHLD Workload Tool}

Eight (57\%), rated the MHLDWT as practical, easy to use, and suitable for calculating staffing requirements in their clinical area. Eleven (79\%) respondents agreed that when balanced against their professional judgement the $M H L D W T$ is a valuable resource for workforce planning. While some participants suggested the MHLDWT was 'quite straight forward', qualitative comments revealed that the majority of participants found it timeconsuming and more difficult to use than the SNCT. This was predominantly due to issues with data collection and entry. 'It was not clear how to realistically collect all the data without following each staff member around. I am not reassured they have truly captured every aspect of care they deliver, despite my advice and encouragement'. Additional criticisms regarding data entry were highlighted for the 'patient specific task' tab. It was suggested that the level of care a person requires fluctuates so quickly, the number of patients for each specific 'task' could alter daily depending on their health'. Finally some respondents suggested that not all tasks associated with the day-to-day running of the ward were compatible with the sections of the tool. These are presented in Table 2. Despite being perceived as time-consuming the majority of respondents suggested the tool provided an accurate representation of their clinical area.

Table 2. A table to illustrate additional tasks associated with the day-to-day running of the ward

\begin{tabular}{|c|c|c|c|}
\hline & Task & & Task \\
\hline \multirow[t]{7}{*}{ Administrative } & Requests for Information & \multirow[t]{7}{*}{ Other } & Staff Meetings \\
\hline & Petty Cash System & & Unplanned Clinician Visits \\
\hline & Return to Work Interviews & & Staff Supervision \\
\hline & Medicines Management & & \\
\hline & Record Keeping (Incident Forms & & \\
\hline & Record Keeping (Spot-checks) & & \\
\hline & Monitoring Emails & & \\
\hline
\end{tabular}




\section{Suitability to different clinical environments}

Qualitative comments revealed some concerns regarding the suitability of both tools to PICU and LD wards. Feedback from PICU wards highlighted the variability of care provided: the bed state is variable throughout the day and often has to be monitored on an hourly basis'. Consequently some respondents suggested that the SNCT was unsuitable as it assumes daily clinical needs remain constant. In addition there were difficulties associated with the use of the MHLDWT on PICU wards. One respondent expressed uncertainty that 'a generic model is suited to this unique mental health environment'. In particular it was suggested that the tasks carried out on a PICU ward did not fit well with the categories in the tool. It was suggested that a way to address this would be to input data that was not directly asked for by the prompt on the system'.

Qualitative comments similarly revealed some concerns regarding the suitability of both tools in LD wards. One respondent suggested that the SNCT appeared to lower the staffing levels of our units'. It was suggested that as it is common for a person with a learning disability to 'require continual support and observation due to their skills level', the workload calculations may not reflect the intensity of care provided. Consequently it was suggested that calculations should be based upon the clinical needs of individual patients as opposed to the needs of the entire ward. Responses from LD wards also revealed difficulties associated with the use of the MHLDWT. It was specifically highlighted that the activities in the 'patient specific' task were too 'generic' and did not fit with this client group e.g. pressure ulcers, some feeding activities, personal hygiene, aseptic dressings, and catheter care.

\section{Workload tool results}

A sample of data returned by participating trusts is presented in Table 3 below. The results highlight inconsistencies between whole time equivalent (WTE) values for Workload and PJ 
calculations. Similarly inconsistencies are evident between Workload calculations for both the MHLDWT and SNCMT for some wards.

Table 3: A table to illustrate a sample of data returned by trusts for both workload measurement tools

\begin{tabular}{|c|c|c|c|c|c|c|}
\hline $\begin{array}{l}\frac{7}{\pi} \\
\frac{\pi}{0} \\
\frac{0}{0} \\
\text { की }\end{array}$ & $\begin{array}{l}\frac{n}{0} \\
\dot{0} \\
0 \\
\dot{2}\end{array}$ & 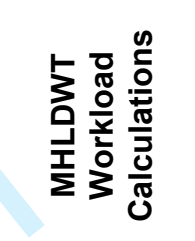 & 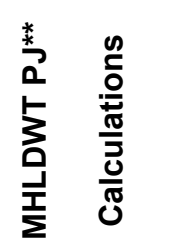 & 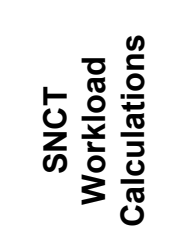 & 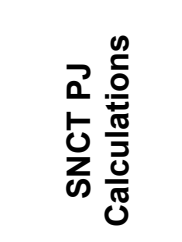 & 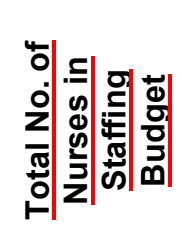 \\
\hline & & ${ }^{*}$ WTE Value & WTE Value & WTE Value & WTE Value & WTE Value \\
\hline Older Adult & 20 & 33.08 & 35.48 & 32.6 & 18.9 & 31.31 \\
\hline Older Adult & 17 & 25.94 & 34.77 & 22.8 & 13.6 & 26 \\
\hline Older Adult & 14 & 50.19 & 29.12 & 31.6 & 22.5 & 23 \\
\hline Adult Acute & 24 & 30.91 & 33.2 & 53.4 & 27.5 & 31.4 \\
\hline Adult Acute & 12 & 22.12 & 32.31 & 12 & 26 & 26 \\
\hline Adult Acute & 20 & 22.62 & 20 & 20.5 & 14 & 20 \\
\hline PICU & 6 & 35.76 & 24.13 & 10.7 & 28.7 & 21 \\
\hline Learning Disability & 10 & 23.74 & 21.18 & 11.7 & 20.8 & 24 \\
\hline
\end{tabular}

*whole time equivalent

${ }^{* *}$ professional judgement

\section{Future use}

The majority of respondents positively viewed the future use of workload measurement tools as a resource to aid nurse staffing calculations at their trust 'overall it was a positive exercise as it provided a framework to look at staffing and I would continue to use them to review/model staffing'. Indeed one respondent suggested the tools outputs highlighted deficits in staffing and resultantly lifted staff morale reassuring staff that they were doing a good job in a busy environment'. Responses highlighted core aspects of care delivery that 
were perceived by users as not being captured by the tools. For example variations in service delivery and structure e.g. '136 assessment suites that are located within units have admission rates and turnover that differ greatly to 'occupied bed days'. Some respondents therefore concluded that the use of evidence-based tools in conjunction with local consultation is essential to the development of workforce plans.

\section{Discussion}

Despite the overall perception that workload measurement tools are a useful resource for workforce planning; the results reveal a number of criticisms regarding the suitability of the SNCT and MHLDWT to mental health and learning disability environments. The SNCT was perceived to be less time consuming and easier to complete; however the results highlight its limited capacity to capture all ward activities due to a predominant focus on the clinical care needs of patients. Contrastingly, criticisms of the MHLDWT were predominantly associated with the complexity of data entry and the resultant time consuming nature of the tool itself. A primary aim of this work was to explore the perceived suitability of the identified staffing calculation tools to complex care settings.

The findings of the study revealed particular concerns regarding the use of the tools in specific care settings. In particular responses from PICU wards suggest the SNCT is unsuitable for use within this care context due to the variability with which care is delivered. This is consistent with research in this area which purports the inability of existing workload calculation systems to adequately account for the fluctuating nature of mental health inpatient environments (Wendling, 2003). Similarly the MHLDWT was criticised for its inability to the capture the range of tasks carried out in this environment. Respondents advocated alterations to both tools in order to accommodate the features of these unique care settings. This suggests a need for further testing of both measures to establish validity 
within specific care contexts and provides an important critique in relation to the use of standardised measures for nurse staffing calculations.

When considering the study findings within the context of international research there was a limited availability of literature with which to make comparisons. Although global challenges such as the 'changing demographics of the ageing population' and higher patient acuity levels are recognised for their impact on nursing workload and staff skill-mix (Gantz et al, 2013, p.435), there has been little to no empirical focus on developing evidence-based staffing levels for mental health and learning disability services. Indeed, Browne et al (2013) in their study of nurse shortages in mental health, report both the lack of research in mental health nursing and the paucity of validated measures to determine the optimal skill-mix of staff. Similarly, Mufaba \& Gates (2014) in their review of literature on LD staffing levels conclude that no empirical work that provides evidence with which to determine safe staffing levels for LD services has yet been undertaken.

This study is the first attempt to capture the perspectives of staff utilising two workload measurement tools that have been adapted for use in MH and LD environments. It therefore has a number of limitations that must be highlighted. Firstly the development of both tools used in this study is on-going; hence reliability and validity has yet to be established. In addition the results show that further testing of the tools across a range of mental health and learning disability environments to review practicability, usability and outcomes is required to determine the perceived suitability of these tools to complex care settings. Furthermore research in this area recommends that the effective implementation of workload measurement tools requires in-depth training for those staff charged with using these tools (Hurst, 2008; Hurst, 2010). Due to the time-constraints associated with this work the training provided was brief. It could therefore be suggested that further training and pro-longed use 
of the tools may yield more positive perceptions from staff utilising these tools within their practice.

\section{Implications for Practice}

Evidence-based tools have an important part to play in the development of workforce planning strategies for mental health and learning disability services. Indeed, the current fiscal climate demands that quality, cost-effective care is provided across all healthcare services. As a result, evidence-based workforce planning for mental health and learning disability clinical environments is vital, particularly given the reducing number of available beds in these settings and increased emphasis on purposeful admission and discharge planning, all of which require appropriate staff levels for delivery. The findings of the study suggest that evidence-based tools for mental health and learning disability inpatient settings require further development. Furthermore, it is essential that staff training highlights awareness about the potential of such tools to enhance inpatient care through effective, evidence-based staffing levels.

\section{References}

Bowers, L. \& Flood, C. (2008). Nurse staffing, bed numbers and the cost of acute psychiatric inpatient care in England. Journal of Psychiatric and Mental Health Nursing. V 15(8), p 630 637.

Brown, V. \& Clarke, V. (2008). Using thematic analysis in psychology. Qualitative Research in Psychology, p. 77 - 101.

Browne, G. Cashin, A., Graham, I., Shaw, W. (2013). Addressing the mental health nurse shortage: Undergraduate nursing students working as assistants in nursing in inpatient mental health settings. International Journal of Nursing Practice, V 19, p. 539 - 545 
Buchan J. \& Aiken L. (2008). Solving nursing shortages: a common priority. Journal of Clinical Nursing, V17, p. $3262-3268$.

Department of Health, (2012). Compassion in Practice: Nursing, Midwifery and Care Staff. Our Vision and Strategy. The Stationery Office, London.

Duffield, C., Roche, M., Diers, D., Catling-Paull, C., and Blay, N. (2010). Staffing, skill mix and the model of care. Journal of Clinical Nursing, V19, p. $2242-2251$.

Francis, R. (2013). Report of the Mid-Staffordshire NHS Foundation Trust Public Inquiry. London: Stationery Office.

Gantz N.R. (2010) 101 Global Leadership Lessons for Nurses: Shared Legacies from Leaders and Their Mentors. Sigma Theta Tau Inc: Indianapolis, IN.

Gantz, N.R., Sherman, R., Jasper, M., Choo, C.G., Herrin-Griffith, D. and Harris, K. (2012). Global nurse leader perspectives on health systems and workforce challenges. Journal of Nursing Management, V20, p. 433 - 443.

Garcia, I., Kennett, C., Quraishi, M. and Durcan, G. (2005) Acute care 2004: a national survey of adult psychiatric wards in England. Sainsbury Centre for Mental Health: London

Happell, B. (2008). Putting all the pieces together: exploring workforce issues in mental health nursing. Contemporary Nurse, V29, p. $43-52$.

Harrison, J. (2004). Applied leadership: addressing increasing patient acuity and workload. 
Nursing Management, V11(4), p. 20 - 25.

Hoekstra T, Lendemeijer H and Jansen M (2004). Seclusion: the inside story. Journal of Psychiatric \& Mental Health Nursing, V 11, p 276-283.

Hurst, K. (2003). Selecting and applying methods for estimating the size and mix of nursing teams. University of Leeds: Nuffield Institute for Health.

Hurst, K. (2008). UK ward design, patient dependency, nursing workload, staffing and quality: an observational study. International Journal of Nursing Studies. V45(3), p. 370 381.

Hurst, K., Smith, A., Casey, A., Fenton, K., Schofield, H., and Smith, S. (2008). Calculating staffing requirements. Nursing Management. V 15(4), p. 26 - 34.

Hurst, K. (2010). Evaluating the strengths and weaknesses of NHS workforce planning methods. Nursing Times. V106, (40), p. 10 - 14.

Kellagher, M., Simpson, J., Flynn, B., Armstrong, D. (2010). Workload and workforce planning: developing a learning toolkit. Nursing Management, V 17(1), p.32 - 34 .

Lockhart, K., Barkby, I., and Kellagher, M. (2010). Workload and workforce planning: taking a national approach. Nursing Management, V 15(9), p. 32 - 34.

Mafuba, K \& Gates, B. (2014). Final report of a systematic review of literature in the public domain on learning disability nursing staffing levels, and its relation to the safety, quality and the delivery of compassionate nursing care. University of West London (unpublished). 
Munro, S., and Baker, J. (2007). Outcomes associated with skill mix interventions in acute mental health wards: a synthesis of evidence. The Journal of Mental Health Training, Education and Practice, V 2(3), p.25 - 33.

National Quality Board, (2013). How to ensure the right people, with the right skills, are in the right place at the right time. A guide to nursing, midwifery and care staffing capacity and capability. London: NHS England.

Spiers, M. (2005). Nurse-patient ratios - a life and death issue. World of Irish Nursing and Midwifery, V 13(3), p. 16.

Ryan, T., Hills, B. \& Webb, L. (2004). Nurse staffing levels and budgeted expenditure in acute mental health wards: a benchmarking study. Journal of psychiatric and mental health nursing, V 11(1), p. $73-81$.

Shelford Group (2014) Safer Nursing Care Tool: Implementation Resource Pack. tinyurl.com/ ShelfordSNCT

Smith, S. (2007), What Contribution Can Acuity Dependency Scoring Make to Nursing Workforce Planning? Manchester Business School, Manchester.

Smith, S., Casey, A., Hurst, K., Fenton, K., Scholefield, H.A. (2009). Developing, testing and applying instruments for measuring rising dependency-acuity's impact on ward staffing and quality. International Journal of Health Care Quality Assurance. V 22(1), p. 30 - 39.

Tummers, G.E., Janssen, P.P., Landeweerd, A. and Houkes, I. (2001). A comparative study 
of work characteristics and reactions between general and mental health nurses: a multisample analysis, Journal of Advanced Nursing, V 36(1), p. 151-62.

Waters, A. (2003). It's all in the mix. Nursing Standard, V 17(23), p. 14-17.

Wendling, L.A. (2003). Clocking care hours with workload measurement tools. Nursing Management, V 34(8), p. 34-39. 